\title{
Divertículos uretrales. Revisión de nuestra casuística y de la literatura
}

\author{
Ramírez Backhaus M, Trassierra Villa M, Broseta Rico E, Gimeno Argente V, \\ Arlandis Guzmán S, Alonso Gorrea M, Jiménez Cruz JF.
}

Servicio de Urología. Hospital Universitario La Fe. Valencia.

Actas Urol Esp. 2007;31(8):863-871

\section{RESUMEN}

\section{DIVERTÍCULOS URETRALES. REVISIÓN DE NUESTRA CASUÍSTICA Y DE LA LITERATURA}

Objetivo: Se valoran los posibles factores etiopatogénicos y se describen la clínica, métodos diagnósticos, abordaje quirúrgico, resultados y complicaciones del manejo de los divertículos uretrales.

Material y métodos: Se realizó una revisión retrospectiva de las historias clínicas con diagnóstico de divertículo uretral en el periodo 1986-2006.

Resultados: Durante estos 20 años se han tratado un total de 19 pacientes con esta patología: 15 mujeres y 4 hombres. Cinco de las mujeres debutaron clínicamente con sensación de masa vaginal; el resto fueron diagnosticadas en el contexto de un síndrome miccional, incontinencia urinaria o infección urinaria. En el caso de los varones, 3 de ellos presentaron una tumoración palpable en el pene. La prueba diagnóstica más utilizada fue la uretrocistografía miccional seriada; la uretrografía con sonda de doble balón se utilizó en 5 ocasiones y la uretroscopia en 4 pacientes. En los casos más complejos se utilizaron otras técnicas de diagnóstico por la imagen, como la resonancia magnética nuclear. El tratamiento fue la extirpación del divertículo en todos los pacientes, excepto en una mujer que rechazó la intervención. La evolución de las pacientes ha sido satisfactoria realizándose controles periódicos hasta dos años después de la intervención. Dos de los casos, en varones, tuvieron recidivas complejas.

Conclusiones: Los divertículos uretrales son una entidad nosológica de difícil diagnóstico dada su baja prevalencia y su clínica inespecífica lo que motiva que, en algunas ocasiones, el diagnóstico sea incidental. La etiopatogenia es adquirida en la mayoría de los casos y su tratamiento quirúrgico, con peores resultados en los hombres debido, probablemente, a que los divertículos se producen en uretras con antecedentes de cirugías previas, manipulación endourológica o lesiones asociadas.

Palabras clave: Uretra. Divertículo. Tratamiento.

\section{ABSTRACT}

\section{URETHRAL DIVERTICULUM. OUR CASUISTIC AND THE LITERATURA REVIEW}

Purpose: The possible etiopathogenic factors, symptoms, diagnostic methods, surgical management and complications of the urethral diverticula are reviewed.

Materials and methods: A retrospective study of the clinical charts with urethral diverticula diagnosis during the period 1986-2006 was carried out.

Results: In the last 20 years a total of 19 patients have been treated for this pathology: 15 females and 4 males. Five of the females started with a sensation of vaginal mass; the rest were diagnosed of micturitional (irritative) syndrome, urinary incontinence or urinary infection. In the case of males, 3 of them had a palpable tumour in the penis. The most used diagnostic method was retrograde and voiding cystourethrography; urethrography with double-occlusion balloon catheter was used in 5 cases and urethroscopy in 4 patients; other techniques of image diagnosis like magnetic resonance imaging were necessary for the most complex cases. The treatment was the excision of the diverticulum, except for one of the females who rejected the treatment. The evolution in all treated women was successful, according to follow up 2 years after the treatment. In males, two of them had complex recurrent diverticula.

Conclusions: Urethral diverticula are nosologic entities of difficult diagnosis, due to their low prevalence and their unspecific clinic, therefore diagnosis is sometimes incidental. The etiopathogenity is acquired in most cases and its surgical treatment is more challenging in males than in females probably linked to the fact that diverticula appear in urethras with previous surgery, endourologic manipulation or associated injuries.

Keywords: Urethra. Diverticulum. Treatment. 
$\mathrm{E}$ 1 divertículo uretral es una dilatación sacular del epitelio uretral en comunicación con la uretra con un origen etiopatogénico adquirido o congénito, y en muchos casos, no aclarado o controvertido.

William Hey describió por primera vez un divertículo uretral femenino en $1805^{1}$. Por aquel entonces la única manera de diagnosticarlo era la exploración física y no fue hasta mediados del siglo XX, cuando, con la generalización de la uretrografía y la uretroscopia, se incrementó la tasa diagnóstica de esta patología.

La incidencia es mayor en mujeres de raza negra $^{2,3}$, el diagnóstico se realiza, generalmente entre la tercera y la quinta década de vida ${ }^{4}$ y la prevalencia global, estimada mediante autopsias o en mujeres que consultaban por otro motivo extraurológico, oscila entre el $0,6 \%{ }^{5}$ y el $4,7 \% \%^{6}$.

Tanto en el varón como en la mujer constituyen una patología infrecuente y la mayoría de las referencias bibliográficas son notas clínicas 0 pequeñas series con 3 ó 4 casos. El manejo de los mismos no está estandarizado ni está referenciado en ninguna guía de práctica clínica. El objetivo de este trabajo de revisión es aportar nuestra experiencia en el manejo de esta entidad y compararlo con el referido en la literatura.

\section{MATERIAL Y MÉTODO}

Se efectúa una revisión retrospectiva y un análisis descriptivo de los casos, estratificados por sexo.

El servicio de urología de nuestro centro ha valorado a 19 pacientes diagnosticados de divertículo uretral en los últimos 20 años. Se trata de 4 hombres y 15 mujeres.

Analizamos los antecedentes medico-quirúrgicos que pudieran estar implicados en la etiopatogenia de esta patología y pormenorizamos los síntomas, signos y hallazgos de la exploración física.

Los métodos diagnósticos utilizados fueron: La cistouretrografía miccional seriada (CUMS), la cistografia con doble balón, la uretrocistoscopia, la urografía intravenosa (UIV), la ecografía transrectal y transvaginal, la tomografía axial computadorizada (TAC) y la resonancia nuclear magnética (RNM).

Las técnicas quirúrgicas realizadas fueron, la diverticulecomía por vía vaginal y la marsupiali- zación en las mujeres, y la diverticulectomía perineal acompañada en algunos casos de uretromía interna o uretroplastia, en los hombres.

Se evaluaron los resultados inmediatos, la evolución y las complicaciones.

\section{RESULTADOS}

En los hombres, dadas sus especiales características, se describen pormenorizadamente.

\section{Caso 1}

Paciente de 56 años con antecedentes de hepatitis $\mathrm{C}$, enolismo, tabaquismo severo y adenocarcinoma de próstata. Dos años antes del diagnóstico se le practicó una prostatectomía radical suprapúbica consecuencia de la cual, el paciente padecía disfunción eréctil e incontinencia urinaria ante esfuerzos leves. Tras objetivarse, al año de la intervención, una recaída bioquímica, se remitió a la unidad de radioterapia donde en una RNM previa al inicio del tratamiento se apreció una tumoración en la base del pene. Posteriormente una Cistouretrografia miccional seriada (CUMS) diagnosticó una estenosis de la anastomosis uretrovesical y un divertículo uretral de $7 \times 5 \mathrm{~cm}$. Se procedió a una diverticulectomía con superposición de tres capas y uretrotomía endoscópica de la estenosis. La evolución fue satisfactoria y el paciente actualmente mantiene, únicamente, una incontinencia urinaria de esfuerzo leve, sin episodios de ITU ni sintomas irritativos.

\section{Caso 2}

Paciente de 50 años con antecedentes de trastorno esquizoide y enolismo que consultó por supuración uretral y masa en la base del pene que aumentaba de tamaño durante la micción. Con la palpación se objetivó una masa empastada en la cara ventral del pene con evacuación de material purulento a la expresión. Se solicitó una CUMS diagnosticando una estenosis de uretra bulbar y un divertículo uretral de $8 \times 5 \mathrm{~cm}$ (Fig. 1), asimismo se solicitó una TC con el fin de planificar mejor el abordaje quirúrgico. Se realizó una uretroplastia término-terminal, diverticulectomía y cierre con interposición de dos capas. A los seis meses de la intervención se objetivó una recidiva del divertículo y dos estenosis de uretra distales al divertículo. Se procedió nuevamente, y previa 


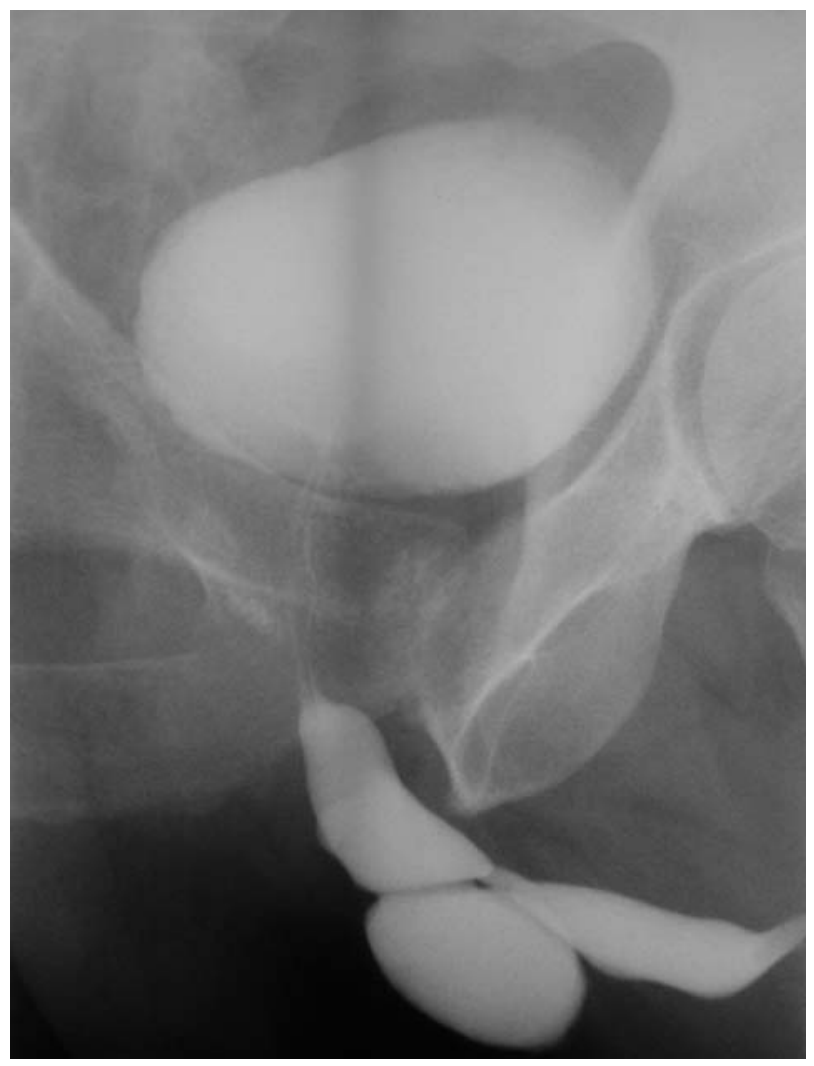

FIGURA 1. Uretrografía miccional retrógrada en la que se objetiva llenado de contraste en una cavidad sacular en uretra bulbar y una estenosis de la uretra distal al diverticulo.

uretrotomía endoscópica de las estenosis a la diverticulectomía. La evolución postoperatoria fue satisfactoria. Con un seguimiento de 12 meses el paciente está asintomático.

\section{Caso 3}

El tercer paciente es un varón de 66 años con antecedentes de diabetes, miastenia gravis, cardiopatía isquémica e infarto agudo de miocardio, que consultaba por una estenosis de la anastomosis uretrovesical tras prostatectomía radical. Tras uretrotomía endoscópica quedó con una incontinencia de esfuerzo severa que motivó la ulterior inyección periuretral de carbono pirolítico. A los 6 meses el paciente consulta por una tumoración dura en la raíz del pene, dolorosa y con salida de material purulento a la expresión. Se realizó una CUMS donde se objetivó el relleno de una cavidad sacular en uretra bulbar correspondiente a un divertículo. Se efectuó una exéresis del divertículo con una evolución satisfactoria, aunque éste recidivó a los dos años. Se reintervino de nuevo extirpando el divertí- culo y cubriendo el defecto uretral con un colgajo longitudinal pediculado de piel de pene. $\mathrm{Al}$ año de esta segunda intervención el paciente presentaba un nuevo divertículo uretral asociado a incontinencia urinaria de esfuerzo. Se le planteó la posibilidad de reintervención con la colocación de un esfinter artificial, pero dada la patología asociada el paciente desestimó la intervención. Con posteridad presentó varias infecciones del trato urinario (ITU), alguna de las cuales requirió ingreso y tratamiento antibiótico endovenoso. A la exploración física se objetivaba una masa dura en el rafe escrotal medio, y en la CUMS y uretroscopia de control se apreciaba un divertículo uretral de mayor tamaño ocupado, ahora, por un cálculo (Fig. 2). Se le planteó nuevamente la cirugía y el paciente está pendiente de intervenir.

\section{Caso 4}

Paciente de 29 años, remitido desde otro centro con importantes antecedentes genitourinarios: agenesia de pubis, onfalocele, atresia anal, hipospadias ya intervenido, estenosis de uretra

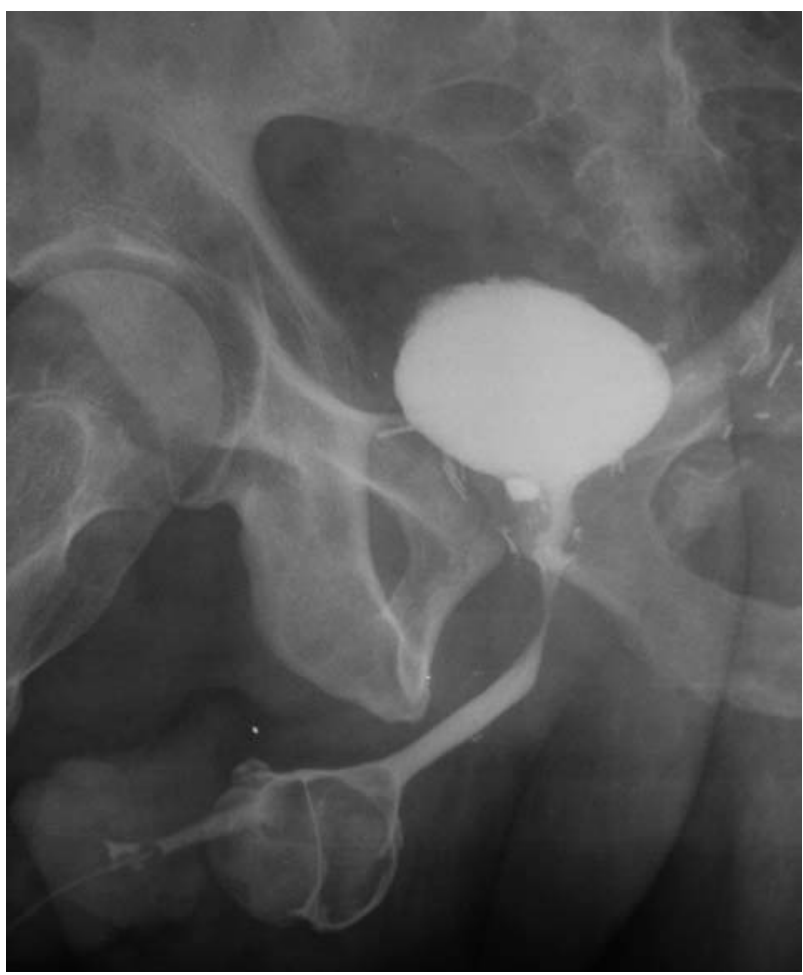

FIGURA 2. Cistografía Miccional Retrógrada donde se rellena una de las cavidades y se objetiva un defecto de repleción en dicha cavidad que se trataba de una litiasis intradiverticular. 
abierta endoscópicamente y litiasis vesical litofragmentada mecánicamente. En la primera anamnesis el paciente refería clínica irritativa intensa y antecedentes de ITU de repetición, alguna de ellas acompañada de fiebre y hematuria. El urocultivo era positivo para E. coli. Como pruebas complementarias se realizaron uretrografía y uretrocistoscopia en las que se objetivó a nivel de la uretra bulbar pre-esfinteriana dos formaciones saculares a derecha e izquierda ocupadas por sendas litiasis. Una ecografia transrectal confirmó la posición parauretral de las litiasis. Una RNM (Fig. 3) no aportó mayor información salvo las alteraciones severas de todas las estructuras anogenitales ya conocidas y la localización de las dos cavidades saculares a ambos lados del rafe medio. Se procedió a una cirugía con resección de ambas cavidades diverticulares y cierre con interposición de tres capas de tejido. Durante la evolución postoperatoria el paciente presentó una infección de la herida quirúrgica que motivó una derivación suprapúbica durante 15 días y una sonda uretral un mes.

Globalmente se han tratado 8 divertículos en 4 pacientes: Un paciente con dos divertículos sincrónicos. En 2 pacientes (50\%) los divertículos recidivaron. En uno de ellos en dos ocasiones. De los 8 divertículos evaluados, 4 (50\%) se acompañaban de estenosis uretral y $3(37,5 \%)$ estaban ocupados por litiasis. Dos pacientes asociaban infecciones urinarias de repetición. Se llevaron a

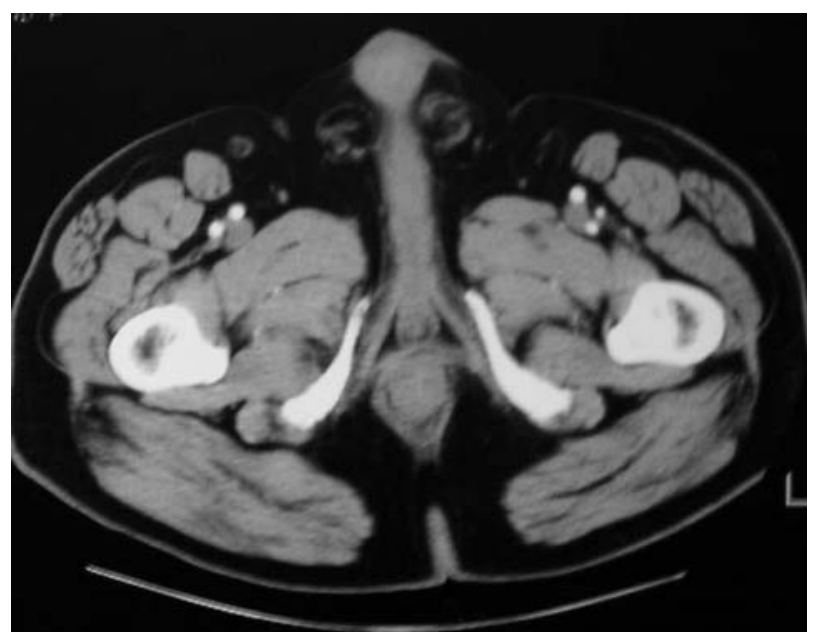

FIGURA 3. TC donde se objetivan a ambos lados del rafe medio dos cavidades parauretrales con contenido heterogéneo en su interior. En esta prueba no se adivina la comunicación con la uretra. cabo 6 intervenciones quirúrgicas y está pendiente de intervenirse el paciente que ha padecido dos recidivas.

Respecto de las mujeres, tratamos a 15 pacientes con una edad media de 46 años y un rango que oscila entre los 30 y 68 años.

Como antecedentes urogenitales de interés objetivamos que a 3 pacientes se les había sometido a una intervención quirúrgica: 1 legrado, una cirugía anti-incontinencia tipo MarshallBurch y otra tercera sufrió un desgarro vaginal por forceps. Cuatro mujeres habían tenido más de 2 partos vaginales eutócicos. Dos pacientes sufrieron una rotura vesical por cesárea, y a una paciente se le había tratado de incontinencia urinaria mediante la inyección de agentes inertes.

Los sintomas que motivaron el diagnostico del divertículo eran inespecíficos: 8 mujeres (53,33\%) consultaban por un sindrome miccional irritativo, $6(40 \%)$ por incontinencia urinaria, $5(33,3 \%)$ por sensación de masa introital, 2 (13,3\%) por ITUs de repetición, $2(13,3 \%)$ por urgencia miccional y $1(6,66 \%)$ por goteo postmiccional.

En cuatro de las pacientes se apreciaba, al tacto vaginal, una masa cuya expresión provocaba la salida de material purulento a través del meato uretral.

La uretrografía con doble balón fue la prueba diagnóstica más utilizada, se le practicó al 60\% de las mujeres; la uretrocistoscopia se realizó en 5 pacientes (33\%), la urografía intravenosa en 5 y una CUMS en 3, la ecografía transvaginal se realizó en dos mujeres. A la mayoría de ellas se les realizó más de una prueba de imagen para el diagnóstico y planificación quirúrgica.

El tratamiento fue quirúrgico en todas excepto en una paciente de 68 años con síntomas que cedieron con una semana de tratamiento antibiótico. Tras ofrecerle una corrección quirúrgica del divertículo, la desestimó. A una de las pacientes se le practicó la marsupialización del divertículo $\mathrm{y}$ al resto una diverticulectomía por vía vaginal. La marsupialización consistió en una incisión longitudinal de la pared anterior de la vagina, liberación del saco diverticular hasta su collarete, apertura y escisión del mismo. El orificio de comunicación uretral en la mayoría de los casos fue cerrado con sutura continua, seguida por un cierre por planos con solapamiento de los bordes 
de la pared vaginal ${ }^{7}$. Tan sólo una de las pacientes presentó un divertículo ocupado por litiasis (6,67\%).

El seguimiento en la mayoría de los casos se llevó a cabo mediante exploración física. A 6 mujeres se les realizó una CUMS en el postoperatorio tardío.

Doce pacientes fueron dadas de alta tras permanecer asintomáticas durante dos años, 3 pacientes presentan ITU de repetición aunque sin haberse apreciado recidiva del divertículo en la CUMS. No se han objetivado otras complicaciones como fistulas, dispareunia o estenosis uretral.

\section{DISCUSIÓN}

Existen en la literatura varios trabajos que han intentado dilucidar la verdadera prevalencia de esta patología. Bruning, en 1959, en 500 autopsias encontró 3 divertículos, lo que supone una frecuencia en este estudio del $0,6 \%{ }^{5}$. En 1967, Andersen realizó un estudio con uretrografía con presión positiva a 300 mujeres sin síntomas del tracto urinario inferior (STUI) en el contexto de un estudio de cáncer de cérvix y objetivó 9 divertículos uretrales, lo que supone una frecuencia para esta serie del $3 \%^{8}$. La mayor incidencia la encuentra Adams que evalúa a 129 mujeres y objetiva 6 divertículos en la uretrografia $(4,7 \%)^{6}$.

Davis y Robison en un trabajo con 972 mujeres de raza negra que consultaban por urgencia miccional, diagnosticaron 58 divertículos uretrales, lo que suponía un a prevalencia en este subgrupo del 6\%; en 5509 mujeres de raza blanca con idéntica clínica objetivaron 62 divertículos, un $1,1 \%^{3}$, Benjamin y cols. describieron 30 casos de divertículos uretrales femeninos de los cuales 19 ocurrian en mujeres de raza negra y 11 en las de raza caucásica ${ }^{2}$. No obstante, en la serie de Leach y Bavendam. de 37 mujeres 20 eran blancas ${ }^{9}$.

El diagnóstico en ocasiones es dificil, y no es hasta la tercera o quinta década de vida, probablemente porque aumenten los controles ginecológicos, cuando es más frecuente el diagnóstico de los mismos ${ }^{9}$.

En la literatura encontramos varias clasificaciones de los divertículos. Según su origen, se pueden dividir en congénitos o adquiridos. Según su localización anatómica, en anteriores o posteriores. Atendiendo a su morfologia se describen como saculares, tubulares, difusos...etc. Los podemos clasificar en auténticos divertículos o pseudodivertículos según conserven o no todas sus capas. Se ha propuesto otra clasificación atendiendo a su morfología endoscópica ${ }^{10}$. En 1993 se propuso una clasificación para divertículos femeninos que consiste en un código de letras: L (en referencia a su localización, distal, media o proximal) $\mathrm{N}$ (en referencia al número) y $\mathrm{S}$ (en referencia al tamaño) y finalmente $\mathrm{C}$ hace referencia a su configuración, comunicación o existencia o no de continencia $^{11}$. Lo cierto es que estas clasificaciones no ayudan en el manejo de esta patología sino que serían meramente didácticas. La mayoría de los autores los diferencia en primarios o secundarios y anteriores o posteriores.

Tanto en el hombre como en la mujer, la etiopatogenia de los divertículos uretrales posteriores suele ser adquirida o iatrógena, sin embargo el origen de los anteriores es más controvertido. El hecho que estén descritos divertículos uretrales en recién nacidos y en mujeres muy jóvenes hace factible la teoría de que algunos divertículos uretrales sean debidos a un cierre defectuoso de los pliegues uretrales primarios o el resultado de la fistulización a uretra de los vestigios del canal de Gartner o de Wolf ${ }^{12}$.

Con los trabajos anatómico-histológicos de Huffman ${ }^{13}$, que describió con detalle la uretra, sus glándulas y conductos adyacentes, se intentó explicar la génesis de los divertículos. En una de las series más extensas de esta patología, 195 de los 204 divertículos se situaban en la cara posterolateral de la uretra entre las tres y las seis horas y en los tercios medial y distal de la uretra ${ }^{14}$; en la teórica localización de las glándulas de Cowper, esto favorece la idea que relaciona los divertículos con la patología de dichas glándulas. Así, quistes congénitos, de retención o alteraciones obstructivas en la desembocadura de estas glándulas, producirian una retención de secreciones, dilatación, y en ocasiones infección, abscesificación hasta el drenaje a la luz uretral y posterior tapizado de la cavidad por epitelio uretral. La presencia de válvulas uretrales anteriores también se ha propuesto como causa de divertículos uretrales anteriores en los hombres ${ }^{15}$. 
Se han descrito como factores etiopatogénicos iatrógenos de los divertículos uretrales posteriores del hombre: La cirugía prostática retropúbi$\mathrm{ca}^{16}$, la prostatectomía radical ${ }^{17}$, la cirugía de las malformaciones anorectales ${ }^{18}$, la inyección transuretral de colágeno ${ }^{19}$. Estos cuatro procedimientos se efectuaron en nuestros pacientes y probablemente fueron los responsables de sus divertículos.

Las lesiones estenóticas, las falsas vías por sondajes, cateterizaciones permanentes, clamps peneanos, colectores, uretritis de repetición y el traumatismo sobre la uretra durante el parto $^{13,19,20}$ también han sido descritos como causa de divertículos uretrales. En definitiva, cualquier proceso que potencialmente interrumpa el flujo urinario normal por la uretra podría favorecer la formación de un divertículo uretral.

La estasis de orina en el divertículo favorece las infecciones urinarias de estos pacientes asî como la precipitación de sales con la consecuente formación de cálculos en su interior, además, la irritación crónica de la orina retenida y contaminada por los cálculos ${ }^{21,22}$ pudiera favorecer la aparición anecdótica de tumores sobre el epitelio que recubre el orificio ${ }^{13,23,24}$.

La clínica que presentan estos pacientes es inespecífica y provocada en ocasiones por las complicaciones del divertículo. Sin embargo, otras veces permanecen totalmente asintomáticos. La palpación de una masa de consistencia más o menos dura, según esté ocupada o no, en el trayecto uretral, con salida de orina o material purulento a la expresión es, para algunos autores, un signo patognomónico. En nuestra serie 2 varones y 4 (sobre 15) mujeres presentan este signo. El cultivo del exudado fue mayoritariamente positivo para bacilos gram negativos.

En las mujeres, la masa aparece en el tabique vaginal anterior ${ }^{23}$. En ellas está descrita una tríada clínica clásica, que no siempre aparece, y que consiste en disuria, dispareunia y goteo posmiccional $^{25}$. En nuestra serie, tan sólo se registra esa clínica en dos de las 15 mujeres. El resto referían síntomas inespecíficos aislados o combinados. Se estima que hasta un $20 \%$ de las mujeres con divertículos uretrales permanecen asintomáti$\operatorname{cas}^{26}$. En nuestro grupo el diagnóstico fue incidental en un hombre y en tres mujeres.
Otros síntomas referidos son los irritativos como consecuencia de infecciones de repetición pese a un adecuado tratamiento antibiótico. La hematuria o la uretrorragia se describen, sobre todo, cuando están acompañados de litiasis intradiverticular. Así pues esta patología, debe incluirse en el diagnóstico diferencial de un paciente con síntomas crónicos del tracto urinario inferior (TUI), o infecciones urinarias de repetición ${ }^{7}$.

Según Romanzi y cols. la media de tiempo transcurrido desde la aparición de los síntomas hasta el diagnóstico suele ser de 5 años, siendo el intervalo de 3 meses a 27 años $^{24}$. En nuestra serie no ha sido factible recoger estos datos pues la mayoría de los pacientes no supo precisar cuando comenzó la clínica o cuando fueron valorados por primera vez.

Tras la sospecha clínica es necesario un diagnóstico de certeza, generalmente es suficiente con técnicas radiológicas con contraste cuyo objetivo es rellenar la cavidad y obtener información del tamaño y posición de la misma. La uretrografia retrógrada y miccional es suficiente hasta en un $60 \%$ de las ocasiones ${ }^{27} \mathrm{y}$, en las mujeres, dada la mayor facilidad de cateterización de la uretra es la prueba de elección utilizando un catéter de doble balón, que aumenta la sensibilidad hasta el $80-90 \%{ }^{28}$. En nuestra experiencia la sensibilidad y especificidad de esta prueba en las mujeres es del $100 \%$. Otra de las técnicas descritas es la punción e inyección de contraste cuando el divertículo se palpa.

La uretroscopia con óptica de 0 grados es útil en la caracterización morfológica del divertículo pudiéndose observar, en ocasiones, la boca del mismo y si está ocupado por litiasis. Se estima que esta prueba tiene una sensibilidad en torno al $60 \%{ }^{29}$, aunque hay series que publican tasas diagnósticas del $100 \% 27$. Es evidente que la rentabilidad de esta prueba dependerá de la experiencia del endoscopista. En nuestra serie se utilizó en 2 hombres y en 5 (33\%) mujeres. El fin no era diagnóstico, sino conocer la distancia exacta del cuello del divertículo hasta el meato y evaluar el estado macroscópico de la mucosa uretral adyacente como parte de la estrategia quirúrgica.

El diagnóstico ecográfico fue introducido por primera vez por Lee y Keller ${ }^{28}$. Actualmente la 
ecografia es transvaginal o transrectal 203031 y es útil en los divertículos posteriores o para diferenciar las masas parauretrales de contenido sólido frente a las líquidas. También detecta la ocupación de un divertículo, cálculos, tumor... etc. La sensibilidad de esta prueba ronda el $30-40 \%{ }^{29}$. No obstante, están descritos falsos negativos de la ecografía transvaginal en aquellos divertículos vacíos, que sólo se llenarían durante la micción ${ }^{29}$ Tabla 1.

Tabla 1. Sensibilidad de las técnicas diagnósticas para divertículos uretrales en mujeres

\begin{tabular}{lc}
\hline Técnica & Sensibilidad \\
\hline Anamnesis y examen físico & $33 \%$ \\
CUMS & $60 \%$ \\
Uretrografía con doble balón & $80 \%$ \\
Uretroscopia & $60 \%$ \\
Ecografía transvaginal & $40 \%$ \\
Ecografía transrectal & $30 \%$ \\
Radiografía postmiccional & $22 \%$ \\
\hline RNM & $70 \%$ \\
\hline
\end{tabular}

La Resonancia nuclear Magnética RNM, otra de las técnicas de imagen útiles para el diagnóstico de esta patología, fue introducida por Kim et al. en $1993^{32}$. A nuestro juicio se debería reservar para los pacientes más complejos, cuando sea preciso conocer la relación del divertículo con los tejidos adyacentes y el estado de los mismos. Robertson planteó un trabajo para comparar la rentabilidad diagnóstica de la uretrografía con doble balón frente a la TC helicoidal sin contraste. Objetivó una mayor sensibilidad de la TC helicoidal y una mayor rentabilidad coste-eficacia. Concluía que sería la prueba de elección ante la sospecha clínica de un divertículo uretral ${ }^{33}$. En nuestra opinión el estudio carece de la potencia estadística necesaria para generalizar sus conclusiones, y en nuestro medio, por el momento, no es aplicabe.

El estudio urodinámico previo al tratamiento podría ser útil para seleccionar aquellas pacientes que se verían beneficiadas de una técnica quirúrgica para la incontinencia asociada junto con la resección del divertículo. Además nos permitiría conocer la máxima presión de cierre uretral y el perfil de presiones uretrales. Probablemente, aunque no hay evidencia al respecto, ayudaría en la elección de la técnica quirúrgica. Dado que, dependiendo de la relación del ostium diverticular con la curva de presiones uretrales se elegiría una u otra técnica: Si el cuello es distal al pico de máxima presión uretral, se podría marsupializar el divertículo, mientras que si es proximal sería más adecuada una diverticulectomía. Bass y Leach en el año 1991 recomendaban el estudio urodinámico en las mujeres con un divertículo uretral con incontinencia urinaria de esfuerzo asociada y les proponían una uretrocistopexia en el mismo acto quirúrgico. Los resultados publicados son buenos ${ }^{34}$. En nuestro centro se solicitaron estudios urodinámicos a 8 de las pacientes que iban a ser intervenidas del divertículo, y en tan sólo un caso se asoció a la exéresis del divertículo la uretrocervicopexia según la técnica de Raz. En otra paciente se colocó un cabestrillo suburetral 10 meses tras la marsupialización del divertículo. A nuestro juicio la presencia del divertículo uretral hace que la curva de presiónflujo del estudio urodinámico sea difícilmente interpretable y no se solicita de forma habitual.

El diagnóstico diferencial de esta patología ha de establecerse con los abscesos de las glándulas de Skene, quistes de inclusión en la pared vaginal, fibromas, miomas, ureteroceles, $\mathrm{u}$ otras masas periuretrales benignas ${ }^{35,36}$.

El abordaje terapéutico de los divertículos uretrales puede ser: conservador o quirúrugico y dentro de ellos; endoscópico o abierto. Se puede optar por un manejo expectante del divertículo en pacientes mayores con un divertículo de pequeño tamaño o asintomático. Está descrito el tratamiento endoscópico con la inyección de agentes inertes como teflón o celulosa aunque la experiencia es escasa y carece de resultados a largo plazo. La apertura endoscópica amplia del cuello diverticular con cuchillete frio o electrocauterio fue descrita por Vergunst et al. ${ }^{37}$ y Lapides ${ }^{38}$ respectivamente.

$\mathrm{El}$ abordaje más frecuente es el abierto y la técnica es diferente en mujeres que en hombres. En las primeras se realiza vía transvaginal y contamos con dos opciones: La marsupialización o la diverticulectomía. La marsupialización se lleva a cabo en aquellos divertículos distales al esfinter 
uretral, es una técnica rápida y sencilla aunque algunas series la han relacionado con altas tasas de ITU o de incontinencia tras la cirugía ${ }^{39}$. La técnica más común es la de Spence que es una forma de meatotomía ampliada hasta el cuello del divertículo ${ }^{40}$.

La diverticulectomía es la técnica más utilizada, aunque la disección de los planos se ve dificultada por el tejido fibrótico o inflamado, que rodea frecuentemente al divertículo, y la presencia de orina residual, a menudo, infectada. El acceso es transvaginal y consiste en una incisión semicircular de la mucosa vaginal, disección y exéresis del divertículo, reconstrucción de la anatomía normal de la uretra e interposición de la mayor cantidad de planos posible, uretra, fascia y colgajo mucoso vaginal. Otra de las dificultades viene dada cuando se extirpa un divertículo de gran tamaño y existe un importante defecto mucoso que precisa la creación de dos colgajos de mucosa vaginal que permitan cerrar el defecto sin tensión.

Cuando un divertículo uretral se infecta y acaba fistulizando a vagina tras la diverticulectomía sería conveniente reforzar la cara posterior de la uretra con un colgajo de labio mayor según la técnica de Martius ${ }^{41}$.

En el caso de los hombres la técnica es más difícil y los resultados más pobres, al no disponer de un tejido de soporte tan versátil como la mucosa vaginal. Además, como se aprecia en nuestra serie, a menudo se asocian una mucosa uretral lesionada por intervenciones previas o por patología asociada. Generalmente se opta por la diverticulectomía abierta.

Las complicaciones más frecuentes de estas intervenciones son la incontinencia urinaria de esfuerzo(1,7-12\%) ${ }^{42,43}$, la recidiva (3\%) o la fístula uretrovaginal (4\%) o uretrocutánea ${ }^{42}$. Las recidivas en mujeres, se abordan mediante una uretrolisis completa y la reconstrucción en un segundo tiempo, y para las fístulas, la interposición de tejido sano como se ha descrito previamente. En los hombres el abordaje se hace cada vez más dificultoso y hay que valorar cada paciente de forma individualizada, teniendo presente las intervenciones en dos tiempos y utilizando siempre una técnica sencilla.

\section{CONCLUSIONES}

Una anamnesis detallada nos ayudará a deducir si se trata de un divertículo adquirido o si existe la posibilidad de que sea congénito.

Cuando, en la exploración física, se detecta una masa en el teórico trayecto uretral con salida de orina o líquido purulento a la expresión de la misma, acompañada de ITU de repetición o síntomas irritativos, existe una alta sospecha de divertículo uretral.

El diagnóstico clínico debe ratificarse con una técnica de diagnóstico por la imagen que, a nuestro juicio, por su alta sensibilidad, especificidad y eficiencia debería de ser la CUMS en el varón y la uretrografía retrógrada a presión positiva con catéter de doble balón en la mujer completando el estudio en algunos casos con una técnica inocua como la ecografía. El resto de pruebas diagnósticas quedarían reservadas a los casos más complejos.

El tratamiento será predominantemente quirúrgico optando por la marsupialización para los divertículos infectados distales al esfinter en mujeres mayores. En el resto de los casos, tanto en hombres como en mujeres el tratamiento de elección será la diverticulectomía con la técnica más simple posible.

$\mathrm{El}$ tratamiento de los divertículos uretrales en los hombres tiene peores resultados. Será fundamental una esterilización previa de la orina con tratamiento antibiótico y una adecuada técnica quirúrgica. Actualmente pensamos que no está justificado un tratamiento de la incontinencia urinaria durante el mismo acto quirúrgico de la exéresis del divertículo, excepto cuando se asocia una incontinencia urinaria de esfuerzo y la paciente demande su resolución.

\section{REFERENCIAS}

1. Hey W. Practical observations in surgery. J Humphreys. Philadelpha 1805.

2. Benjamin J, Elliott L, Cooper JF, Bjornson L. Urethral diverticulum in adult female. Clinical aspects, operative procedure, and pathology. Urology. 1974;3(1):1-7.

3. Davis BL, Robinson DG. Diverticula of the female urethra: assay of 120 cases. J Urol. 1970;104(6):850-853.

4. Leach GE, Bavendam TG. Female urethral diverticula. Urology 1987;30(5):407-415.

5. Bruning EJ. Die pathologie der weisslichen urethra und des parurethrium. Enke. 1959.

6. Andersen MJ. The incidence of diverticula in the female urethra. J Urol. 1967;98(1):96-98. 
7. Adams WE. Urethrography. Bull Tulane Univ Med Fac. 1964;23:107-112.

8. Chinesta SS, Gorrea MA, Martínez Jabaloyas J, Martínez Sarmiento M, Jiménez Cruz JF. Diverticulum of the female urethra. Review of the literature. Actas Urol Esp. 1994; 18(9):871-879.

9. Valdivia Uria JG, Lopez Lopez JA, Aranda Lassa JM, Romero Aguirre F. Bulbourethral diverticuli]. Arch Esp Urol. 1986;39(2): 117-32.

10. Leach GE, Sirls LT, Ganabathi K, Zimmern PE. L N S C3: a proposed classification system for female urethral diverticula. Neurourol Urodyn. 1993;12(6):523-531.

11. De Diego Rodriguez E, Martin Garcia B, Hernandez Rodriguez R, Portillo Martin JA, Cqrreas Gomez MA, Gutierrez Banos JL, et al. Urethral diverticula in the male. Our experience over 10 years. Arch Esp Urol. 1998;51(9): 859-63.

12. Huffman JW. The detailed anatomy of the par. Am J Obstet Gynecol. 1948;55:86-88.

13. Mackinnon M, Pratt JH, Pool TL. Diverticulum of the female urethra. Surg Clin North Am. 1959;39(4):953-962.

14. Moskowitz PS, Newton NA, Lebowitz RL. Retention cysts of Cowper's duct. Radiology. 1976;120(2):377-80.

15. Orejas Lopez V. Female urethral diverticuli. Arch Esp Urol. 2002;55(9):1137-1143.

16. Currarino G, Fuqua F. Cowper's glands in the urethrogram. Am J Roentgenol Radium Ther Nucl Med. 1972; 116(4):838-842.

17. Gil-Vernet S. "Divertículos bulbouretrales y reflejo vesicouretral”. Arch Esp Urol. 1977;30:325.

18. Mansson W, Colleen S, Holmberg JT. Cystic dilatation of Cowper's gland duct -an overlooked cause of urethral symptoms? Scand J Urol Nephrol. 1989;23(1):3-5.

19. Williams DI, Retik AB. Congenital valves and diverticula of the anterior urethra. Br J Urol. 1969;41(2):228-234.

20. England EJ, Low AI. Pseudodiverticulum of prostatic urethra after retropubic prostatectomy. Urology. 1975;6 (3):348-350.

21. Laudone VP, Green KF, Wyker AW, Jr. Giant posterior urethral diverticulum after radical retropubic prostatectomy. J Urol. 1988;139(2):364-366.

22. Vinnicombe SJ, Good CD, Hall CM. Posterior urethral diverticula: a complication of surgery for high anorectal malformations. Pediatr Radiol. 1996;26(2):120-126.

23. Clemens JQ, Bushman W. Urethral diverticulum following transurethral collagen injection. J Urol. 2001;166(2):626.

24. Pamplona M, Paniagua P, García-Castaño B, Avila E, Extramiana J, González P, et al. Urethral diverticulum in women. Echographic diagnosis. Actas Urol Esp. 1990;14(4):252-257.

25. Andreu A LM. Litiasis intradiverticular en uretra femenina. A propósito de un caso. Actas Urol Esp. 1992;16:269.

26. Guidi HG, Montelatto NI, Ribeiro RM, Pinotti JA. The treatment of female urethral diverticulum with calculus through ultrasonic lithotripsy. Int $J$ Gynaecol Obstet. 1993;41(3): 277-281.

27. Ponce Campuzano A, Colom Feixas S, Matias Garcia JJ, Franco Miranda E, Orejas Lopez V, Serrallach Mila N. Diverticulum of the female urethra: presentation of 3 cases. Actas Urol Esp. 1998;22(2):154-158.

28. Romanzi LJ, Groutz A, Blaivas JG. Urethral diverticulum in women: diverse presentations resulting in diagnostic delay and mismanagement. J Urol. 2000;164(2):428-433.

29. Butler WJ. The diagnosis of urethral diverticula in women. J Urol. 1966;95(1):63-64.
30. Palagiri A. Urethral diverticulum with endometriosis. Urology. 1978;11(3):271-272.

31. Summitt RL, Stovall TG. Urethral diverticula: evaluation by urethral pressure profilometry, cystourethroscopy, and the voiding cystourethrogram. Obstet Gynecol. 1992;80(4): 695-699.

32. Lee TG, Keller FS. Urethral diverticulum: diagnosis by ultrasound. AJR Am J Roentgenol 1977;128(4):690-1.

33. Davis HJ, Cian LG. Positive pressure urethrography: a new diagnostic method. J Urol 1956;75(4):753-7.

34. Greenberg M, Stone D, Cochran ST, Bruskewitz R, Pagani JJ, Raz S, et al. Female urethral diverticula: double-balloon catheter study. AJR Am J Roentgenol. 1981;136(2): 259-264.

35. Kohorn EI, Glickman MG. Technical aids in investigation and management of urethral diverticula in the female. Urology. 1992;40(4):322-325.

36. Fortunato P, Schettini M, Gallucci M. Diagnosis and therapy of the female urethral diverticula. Int Urogynecol $\mathrm{J}$ Pelvic Floor Dysfunct. 2001;12(1):51-57.

37. Baert L, Willemen P, Oyen R. Endovaginal sonography: new diagnostic approach for urethral diverticula. J Urol. 1992;147(2):464-466.

38. De Simone G CR, Martino D. Diagnostic echographique d'un cas rare de diverticule de l'uretre posterieur. J Urology. 1998;4:233.

39. Kim B, Hricak H, Tanagho EA. Diagnosis of urethral diverticula in women: value of MR imaging. AJR Am J Roentgenol. 1993;161(4):809-815.

40. Robertson J. Urethral diverticula. 3 ed: Baltimore: Wiliams and Wilkins, 1991.

41. Bass JS, Leach GE. Surgical treatment of concomitant urethral diverticulum and stress incontinence. Urol Clin North Am. 1991;18(2):365-373.

42. Boyd SD, Raz S. Ectopic ureter presenting in midline urethral diverticulum. Urology. 1993;41(6):571-574.

43. Dmochowski RR, GK, Zimmern PE. Bening female periurethral masses. J Urology. 1994;152:1953.

44. Vergunst H, Blom JH, De Spiegeleer AH, Miranda SI. Management of female urethral diverticula by transurethral incision. Br J Urol. 1996;77(5):745-746.

45. Lapides J. Transurethral treatment of urethral diverticula in women. J Urol. 1979;121(6):736-738.

46. Lichtman AS, Robertson JR. Suburethral diverticula treated by marsupialization. Obstet Gynecol. 1976;47(2):203-206.

47. Spence HM, Duckett JW. Diverticulum of the female urethra: clinical aspects and presentation of a simple operative technique for cure. J Urology. 1970;104(3):432-435.

48. Webster GD SS, Stone AR. Urethrovaginal fistula. A reviewof the surgical managment. J Urology. 1984;132:460464.

49. Ward JN, Draper JW, Tovell HM. Diagnosis and treatment of urethral diverticula in the female. Surg Gynecol Obstet. 1967; 125(6): 1293-300.

50. Wharton GD SS, Stone AR. Urethral diverticulum. Obstet Gynecol. 1956;7:503-506.

Correspondencia autor: Dr. Miguel Ramírez Backhaus

Servicio de Urología. Hospital Universitario La Fe

Avda. Campanar, 21 - 46009 Valencia

E-mail autor: ramirezbackhaus@yahoo.es

Tel.: 963862700

Información artículo: Original - Urología femenina

Trabajo recibido: octubre 2006

Trabajo aceptado: abril 2007 